WILEY-VCH

\title{
3D structure determination of an unstable transient enzyme intermediate by paramagnetic NMR spectroscopy
}

\author{
Jia-Liang Chen ${ }^{\mathrm{a}}$, Xiao Wang ${ }^{\mathrm{a}}$, Feng Yang ${ }^{\mathrm{a}}$, Chan Cao ${ }^{\mathrm{a}}$, Gottfried Otting ${ }^{\mathrm{b}}$, and Xun-Cheng Su*a
}

\begin{abstract}
Enzyme catalysis of chemical reactions relies on conformational plasticity but structural information on transient intermediates is difficult to obtain. Here we show that the threedimensional (3D) structure of an unstable, low abundance enzymatic intermediate can be determined by nuclear magnetic resonance (NMR) spectroscopy. The approach is demonstrated for Staphylococcus aureus sortase A (SrtA), which is an established drug target and biotechnological reagent. SrtA is a transpeptidase that converts an amide bond of substrate peptide to a thioester. By measuring pseudocontact shifts (PCS) generated by a site-specific cysteine-reactive paramagnetic tag that does not react with the active site residue Cys184, a sufficient number of restraints could be collected to determine the $3 \mathrm{D}$ structure of the unstable thioacyl intermediate of SrtA that is present only as a minor species under nonequilibrium conditions. The 3D structure reveals structural changes that protect the thioacyl intermediate against hydrolysis and indicate a positive allosteric effect between the thioester bond and a calcium binding site.
\end{abstract}

Delineating conformational changes of protein enzymes at atomic resolution is required for a detailed understanding of their functions..$^{1,2}$ Different methods for structural characterization of enzymes during chemical reaction have been developed recently. ${ }^{1,2,3}$. Structure determinations of unstable enzyme intermediates in solution, however, are still a challenge in view of the short lifetime and low abundance of intermediates, which makes it difficult to collect sufficient structural restraints in the time available. Recent advances in NMR spectroscopy have made it possible to obtain protein structures from NMR data other than nuclear Overhauser effects, such as chemical shifts, residual dipolar couplings (RDC), paramagnetic relaxation enhancement (PRE), and pseudocontact shifts (PCS). 4-12 PCSs stand out for providing localization restraints for nuclear spins relative to the paramagnetic center that can be measured in minutes in sensitive heteronuclear single quantum coherence (HSQC) spectra. Here we show how PCSs allowed the 3D structure determination of the unstable transient thioacyl intermediate formed by Staphylococcus aureus Sortase A (SrtA) and a peptide substrate. SrtA is an important protein enzyme in Gram-positive bacteria. It converts a backbone amide of substrate peptide (the peptide bond between threonine and glycine in polypeptides containing

[a] Dr J. L. Chen, Ms X. Wang, Ms C. Cao, Mr. F. Yang, Prof. X. S. Su State Key Laboratory of Elemento-Organic Chemistry

Collaborative Innovation Center of Chemical Science and Engineering (Tianjin), Nankai University, Tianjin 300071, China E-mail: xunchengsu@nankai.edu.cn

[b] Prof. G. Otting

Research School of Chemistry, Australian National University, Canberra, ACT 2601, Australia

Supporting information for this article is given via a link at the end of the document. the LPXTG motif, where $X$ can be any amino acid) into a thioester with the active-site cysteine residue (Cys184). ${ }^{13-16} \mathrm{SrtA}$ is an established drug target ${ }^{15}$ and is increasingly being used as a biotechnological tool for protein ligations. ${ }^{17,18}$ 3D structures of SrtA have been determined by X-ray crystallography and NMR spectroscopy. ${ }^{19,20}$ The structure of disulfide bond linked thioacyl analogue SrtA-LPAT* has been solved by NMR and showed significantly different structural features compared with free SrtA or SrtA in a non-covalent complex with peptide substrate. ${ }^{21}$ While the occurrence of a SrtA thioacyl complex can be detected in a mass spectrum, ${ }^{22,23}$ but the $3 \mathrm{D}$ structure of this unstable intermediate has not been determined. Thioacyl complexes are intermediates in many enzyme reactions,$^{24}$ making techniques for their structural elucidation highly desirable.

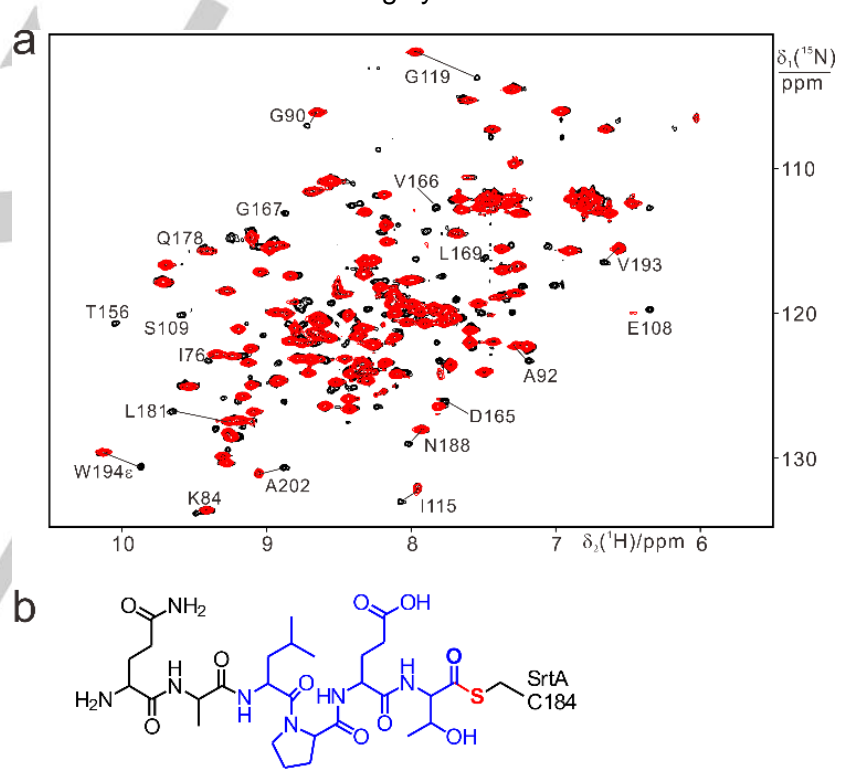

Figure 1. NMR detection of the SrtA thioacyl intermediate during hydrolysis of substrate peptide. All ${ }^{15} \mathrm{~N}-\mathrm{HSQC}$ spectra were recorded of $0.1 \mathrm{mM}$ solutions of uniformly ${ }^{15} \mathrm{~N}$-labeled SrtA in $20 \mathrm{mM}$ MES buffer, $\mathrm{pH} 6.4$, at $298 \mathrm{~K}$ in the presence of $1.0 \mathrm{mM} \mathrm{CaCl}_{2}$. a) Superimposition of ${ }^{15} \mathrm{~N}-\mathrm{HSQC}$ spectra before (red) and after (black) mixing with $1.0 \mathrm{mM}$ peptide QALPETG-NH . Corresponding cross-peaks of the thioacyl intermediate (minor species) and free SrtA (major species) from the same residues are connected by lines. b) Chemical structure of the thioacyl intermediate formed between SrtA and QALPETG-NH .

In the present work, we identified conditions under which SrtA (without the $\mathrm{N}$-terminal 58 amino acids that serve as a membrane anchor in full-length SrtA) ${ }^{19}$ transiently displays resolved NMR resonances for the thioacyl intermediate. We next labeled SrtA site-specifically with a lanthanide binding tag and measured PCSs of the short-lived thioacyl intermediate formed between Cys 184 of SrtA and the threonine residue of the substrate peptide QALPETG. 
Using the PCSs as structural restraints, we determined the 3D structure of the thioacyl intermediate state of SrtA.

a

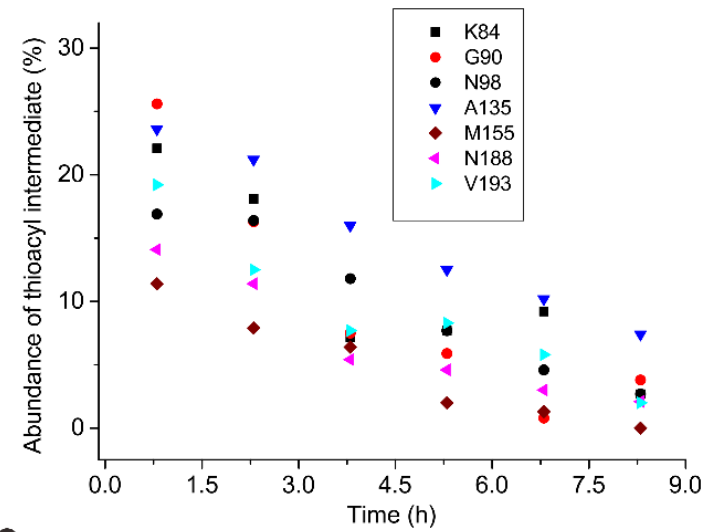

b

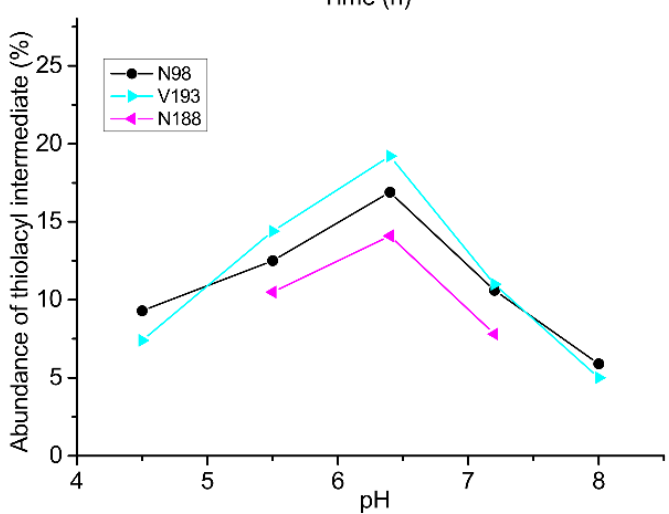

C

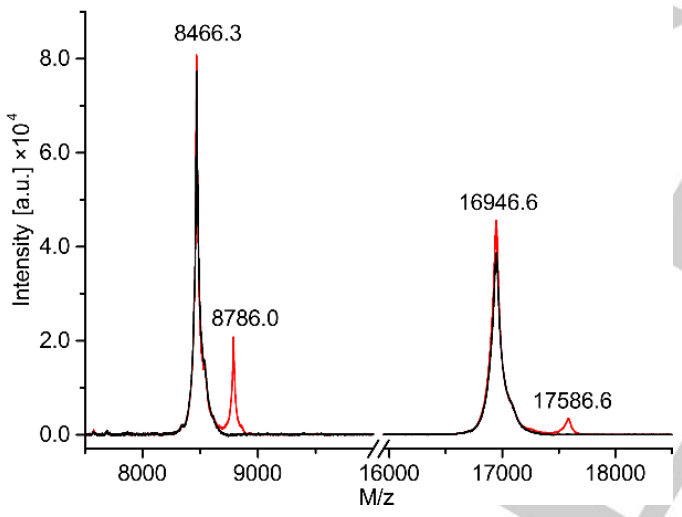

Figure 2. Population of thioacyl intermediate. a) Cross-peak volumes of selected residues of the thioacyl intermediate relative to the sum of cross-peak volumes of minor and major species, which provides an estimate of the population of the thioacyl species, as a function of incubation time. Different cross-peak amplitudes between different residues result from different line widths in the minor and major species. Apparent variations in decay rates arise from limited sensitivity of weak cross-peaks. b) Abundance of the thioacyl intermediate as a function of $\mathrm{pH}$ determined from ${ }^{15} \mathrm{~N}$-HSQC cross-peak volumes as in a) (see supporting material). c). MALDI-TOF mass spectra recorded for the mixture of $0.1 \mathrm{mM}$ SrtA (unlabeled) and $1.0 \mathrm{mM} \mathrm{Ca}^{2+}$ prepared without (black) and with (red) $1.0 \mathrm{mM}$ QALPETG-NH $\mathrm{N}_{2}$ in $20 \mathrm{mM}$ MES buffer at $\mathrm{pH}$ 6.4. The calculated molecular mass difference of 640 mass units between SrtA and thioacyl SrtAQALPET is identical to the difference of masses observed (16946.6 and 17586.6).<smiles>O=C(O)CN(CC(=O)O)Cc1cc(SSc2cc(CN(CC(=O)O)CC(=O)O)nc(CN(CC(=O)O)CC(=O)O)c2)cc(CN(CC(=O)O)CC(=O)O)n1</smiles>

$\mathrm{T} 1$ $\mathrm{T} 2$

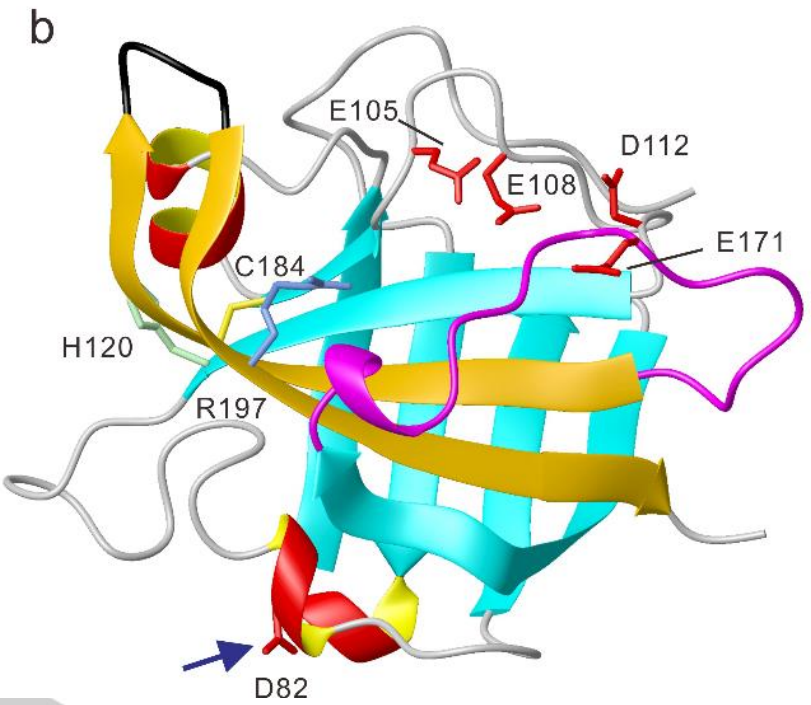

Figure 3. Site-specific labeling of SrtA D82C with a paramagnetic lanthanide tag. a) Chemical structures of the tags $\mathrm{T} 1$ and $\mathrm{T} 2$ used in the present study. b) Ribbon representation of the SrtA structure (PDB code: $1 \mathrm{~T}^{2} \mathrm{P}^{20}$ ), highlighting the active site and calcium binding site by showing the side chains of catalytically important residues (His120, Cys184, and Arg197) and of residues involved in calcium binding (Glu105, Glu108, Asp112, and Glu171), respectively. The strands $\beta 7$ and $\beta 8$ are shown in orange, the preceding $\beta 6 / \beta 7$ loop in magenta, and the $\beta 7 / \beta 8$ loop in black. The arrow points at the side chain of Asp82 that was mutated to cysteine for paramagnetic tagging.

NMR signals of the intermediate were identified by monitoring the reaction of SrtA with substrate peptide (QALPETG$\mathrm{NH}_{2}$, where $\mathrm{NH}_{2}$ serves as a protection group at the C-terminal glycine) by ${ }^{15} \mathrm{~N}-\mathrm{HSQC}$ spectra. Many new weak cross-peaks appeared (Figure 1a), which decreased with a half-life of about five hours (Figure 2a) and were most highly populated at $\mathrm{pH} 6.4$ (Figure 2b). Mass spectrometry indicated that the new species was the thioacyl intermediate (Figure 2c). ${ }^{22,23}$ The intermediate could not be detected in the absence of calcium or in the presence of $1.0 \mathrm{mM}$ tri-glycine peptide, which is known to act as a second substrate to resolve the thioester by forming a peptide bond with the bound first substrate (Figures S1 and S2).

The lifetime and concentration of the thioacyl complex was too short for NMR resonance assignments by standard 3D tripleresonance experiments. Therefore, we assigned the resonances by comparison with a stable disulfide-bonded analogue produced by ligating the peptide QALPECG-NH $\mathrm{NH}_{2}$ to Cys184 of SrtA (Scheme S1). ${ }^{25}$ The ${ }^{15} \mathrm{~N}-\mathrm{HSQC}$ spectrum of the disulfide bond linked SrtA-QALPECG-NH $\mathrm{N}_{2}$ adduct showed cross-peaks at very similar chemical shifts as the SrtA-QALPET thioacyl complex in the presence of $\mathrm{Ca}^{2+}$ (Figure S3) and the backbone assignment of the SrtA-QALPECG-NH $\mathrm{N}_{2}$ adduct was readily obtained by a 3D 
NOESY- ${ }^{15} \mathrm{~N}-\mathrm{HSQC}$ spectrum. Residues showing large chemical shift differences between the SrtA-QALPECG- $\mathrm{NH}_{2}$ adduct and the thioacyl intermediate mainly involved amides close to Cys 184 , reflecting the chemical difference between the disulfide and thioester bond linkages. Similarly, the calcium binding motif (Glu105, Glu108, Asp112, Asn114, and Glu171)26 displayed almost identical chemical shifts in the ${ }^{15} \mathrm{~N}-\mathrm{HSQC}$ spectra of the SrtA-QALPECG- $\mathrm{NH}_{2}$ adduct and thioacyl intermediate. Judging by the chemical shift differences, the structural difference to free SrtA is much greater (Figure S3).

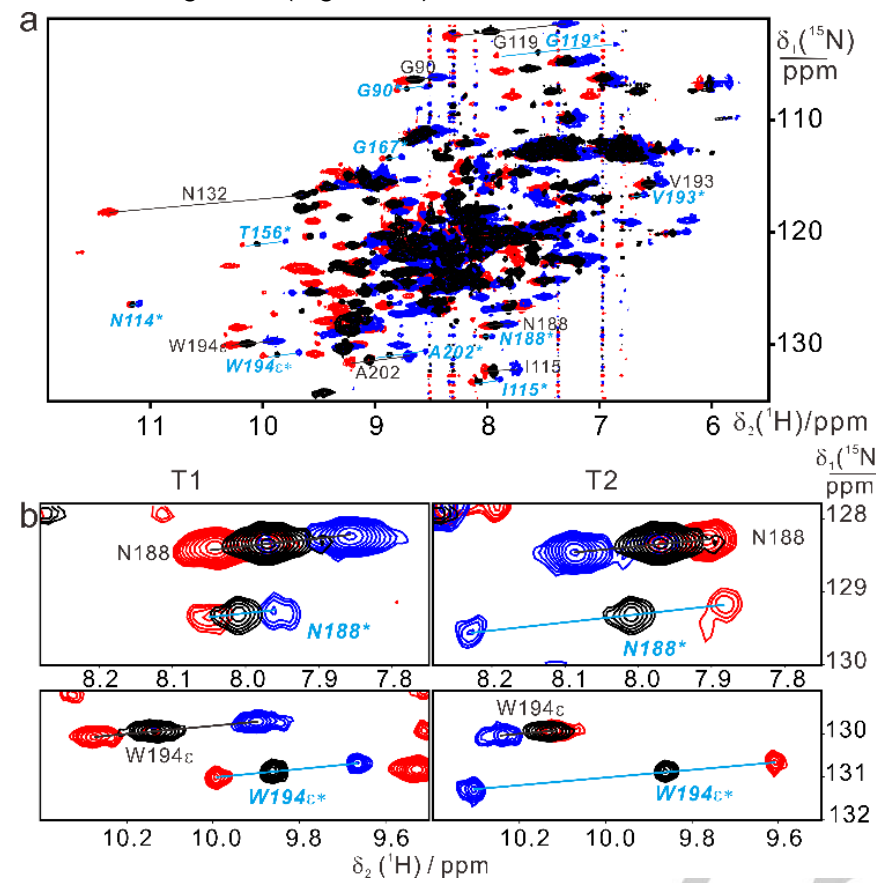

Figure 4. PCSs measured for SrtA D82C labeled with the lanthanide binding tags T1 or T2. Superimpositions of ${ }^{15} \mathrm{~N}-\mathrm{HSQC}$ spectra measured of $0.15 \mathrm{mM}$ solutions of tagged SrtA D82C in complex with one equivalent of $\mathrm{Y}^{3+}$ (black), $\mathrm{Tm}^{3+}$ (red), or $\mathrm{Tb}^{3+}$ (blue). Selected cross-peaks are labeled with their resonance assignment, using black labels for the free protein, and blue labels and stars for the corresponding cross-peaks of the thioacyl intermediate. a) Spectra recorded of $0.15 \mathrm{mM}$ SrtA D82C with T1 tag in the presence of $1.0 \mathrm{mM}$ $\mathrm{Ca}^{2+}$ and $1.0 \mathrm{mM}$ QALPETG-NH $\mathrm{H}_{2}$. b) Zoom into selected regions of the ${ }^{15} \mathrm{~N}$ HSQC spectra recorded of the thioacyl intermediate, highlighting differences in the PCSs for the thioacyl intermediate and the free protein. Left panel: ${ }^{15} \mathrm{~N}$ HSQC spectra of SrtA D82C with T1 tag shown in b). Right panel: same as b), except that the spectra were recorded for SrtA D82C with T2 tag.

Following assignment of the ${ }^{15} \mathrm{~N}-\mathrm{HSQC}$ spectrum, we sought to collect structural restraints of the thioacyl intermediate. Most of the ${ }^{15} \mathrm{~N}$-HSQC cross-peaks of the thioacyl intermediate were sufficiently well resolved to enable accurate PCS measurements of protein samples that were site-specifically labeled with a paramagnetic ion. As SrtA contains a $\mathrm{Ca}^{2+}$ binding motif and $\mathrm{Ca}^{2+}$ is important for enhancing the enzymatic activity, ${ }^{19}$ any paramagnetic lanthanide tag needs to have a higher binding affinity for lanthanide than calcium ions. Moreover, the thiol group of Cys 184 could potentially react with and be inactivated by a cysteine-reactive lanthanide tag. To address these constraints, we made the $\mathrm{D} 82 \mathrm{C}$ mutant of SrtA and synthesized the new paramagnetic tags T1 and T2 (Figure 3a; see the Supplementary
Schemes S2-S5 for tag synthesis and ligation with SrtA). T1 reacts with a solvent-exposed thiol group in aqueous solution at $\mathrm{pH} 7.6$ with the release of phenylsulfonate as the leaving group and, owing to the bromine atom, is more reactive than the previously published $4 \mathrm{PhSO}_{2}$-PyMTA tag. ${ }^{27} \mathrm{~T} 2$ reacts with a protein thiol similar to 4,4-dithiobisdipicolinic acid with formation of a disulfide bond. ${ }^{28}$ Both tags showed high chemoselectivity towards the thiol group of the cysteine at position 82, but were not reactive towards the side chain of Cys184, as confirmed by chemical shift mapping and MALDI-TOF mass spectra (Figures S4 and S5). The tags have high affinities for lanthanide ions, which were not compromised by a large excess of $\mathrm{Ca}^{2+}$ (up to 20 equivalents).

To generate the paramagnetically tagged thioacyl intermediate, solutions of the SrtA D82C-T1/T2 adducts were first titrated with lanthanides ions in the presence of calcium, which maintained saturation of the calcium binding site with $\mathrm{Ca}^{2+}$ (Figures $3 \mathrm{a}$ and S6). Subsequent addition of QALPETG-NH peptide generated the thioacyl intermediate. As expected, only two paramagnetic species were observed (Figures 4a and S6), where the major species corresponded to the free SrtA D82C$\mathrm{T} 1 / \mathrm{T} 2$ complexes, while the minor species corresponded to the thioacyl intermediates (Figure 4b). PCSs were determined from the ${ }^{1} \mathrm{H}$ chemical shift differences between the paramagnetic species, generated with $\mathrm{Tm}^{3+}$ or $\mathrm{Tb}^{3+}$ ions, and the diamagnetic species, using $\mathrm{Y}^{3+}$. Different PCSs were observed for the backbone amide protons of the thioacyl intermediate and of free SrtA (Figures 4a, 4b, S6, and S7).

Using the program Numbat, ${ }^{29}$ the magnetic susceptibility anisotropy $(\Delta \chi)$ tensors of the lanthanide complexes were determined using PCSs and the crystal structure of free SrtA (PDB code: $1 \mathrm{~T}^{2} \mathrm{P}^{20}$ ), using only protein segments with regular secondary structure, for which neither chemical shifts nor PCSs changed significantly between free SrtA and the thioacyl intermediate. The resulting $\Delta \chi$ tensor parameters are listed in Table S1. Similar $\Delta \chi$ tensors were obtained by using the NMR structure of SrtA (PDB code: $1 \mathrm{IJA}^{19}$, Table S2). The $\Delta \chi$ tensor parameters of the thioacyl intermediate were similar to that of free SrtA, suggesting that formation of the thioacyl intermediate causes no significant structural rearrangement for the structurally well-defined segments of the protein. T1 and T2 tags generated different paramagnetic tensors, providing complementary information for subsequent structure calculations.

Next we determined the structure of the thioacyl intermediate from the PCSs. A total of 407 PCSs (262 PCSs for D82C-T1QALPET, 145 for D82C-T2-QALPET) collected for $\mathrm{Tm}^{3+}$ and $\mathrm{Tb}^{3+}$ ions were used to compute the structure of loop regions in the thioacyl intermediate using the program Xplor- $\mathrm{NIH}^{30-32}$ The conformation of structurally conserved regions of the protein, as judged by conservation of chemical shifts and PCSs, was kept rigid by a set of backbone dihedral angle restraints derived from available structures of SrtA. Starting from the crystal structure of free SrtA (PDB code: 1T2P), ${ }^{20}$ a total of 1000 conformers were calculated (for details see the Supporting Information). Figure $5 \mathrm{a}$ presents the 20 lowest energy conformations out of 1000 structures calculated for the thioacyl intermediate. The calculated structures converged well, with $\mathrm{C} \alpha$ and heavy atom rmsd values to the average structure of 0.7 and $1.1 \AA$, respectively. Starting the calculations from the NMR structure of the disulfide bond 
linked thioacyl analogue, SrtA-LPAT* (PDB code: 2KID) ${ }^{21}$ yielded very similar conformations (Figures S8 and S9, and Tables S3 and S4). Compared with the crystal structure of SrtA, the thioacyl intermediate revealed notable structural changes in several regions, including the $\beta 6 / \beta 7$ and $\beta 7 / \beta 8$ loops (Figure 5 ). The residues in these regions are important for catalytic function and were found to undergo conformational exchange in earlier NMR studies of free SrtA..$^{20,21}$ In addition, residues in the first helix ( $\left.\alpha 1\right)$ and the $\mathrm{Ca}^{2+}$ binding motif, including the loop segments $\beta 3 / \beta 4$, $\beta 4 / \alpha 2$, and $\beta 5 / \beta 6$, also showed significant structural changes.
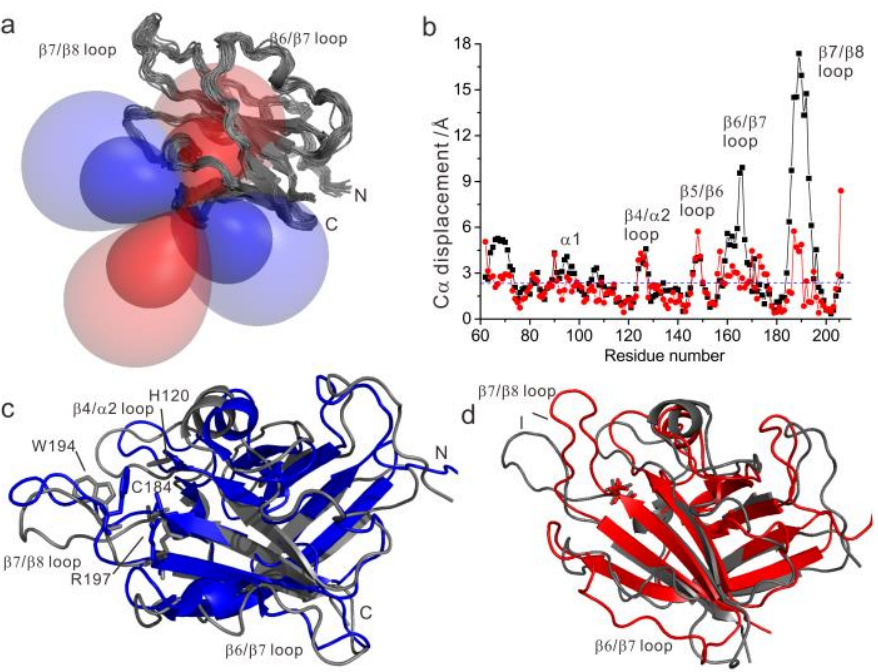

Figure 5. 3D structure of the thioacyl intermediate defined by PCSs. a) Superimposition of the 20 lowest energy structures of the thioacyl intermediate calculated using the PCSs. PCS isosurfaces of the thioacyl SrtA D82C-T1-Tb complex are shown together with the protein structure. The isosurfaces plotted correspond to PCSs of 0.8 and 0.2 ppm (blue) and -0.8 and -0.2 ppm (red). b) Backbone $\mathrm{Ca}$ displacement of the lowest-energy structure of the thioacyl intermediate relative to the crystal structure of free SrtA (black squares) (PDB code: $1 \mathrm{~T}^{2} \mathrm{P}^{20}$ ) and to the first conformer of the NMR structure of the disulfidelinked thioacyl analogue, SrtA-LPAT* (red spheres) (PDB code: 2KID ${ }^{21}$ ). C) Structure comparison between the thioacyl intermediate (gray) and SrtA-LPAT* (PDB code: $2 \mathrm{KID}^{21}$, blue). The side chains of residues 120,184 , and 197, which form a triangle marking the active site, and of Trp194 are shown as sticks. Loop regions with significant structural differences are labeled. d) Superimposition of sortase A structures of the thioacyl intermediate (gray), SrtA with inhibitor (PDB code: $2 \mathrm{MLM}^{33}$, red)

The structure of the thioacyl intermediate most closely resembles the structure of the disulfide-bonded SrtA-LPAT* analogue (PDB code: $2 \mathrm{KID},{ }^{21}$ Figure $5 \mathrm{~b}$ and $\mathrm{c}$ ) and differs significantly from the crystal structure of SrtA with non-covalently bound LPETG peptide (PDB code: 1 T2W) ${ }^{20}$ which has almost the same conformation as free SrtA. The $\beta 6 / \beta 7$ and $\beta 7 / \beta 8$ loops in the thioacyl intermediate also differ significantly from the structure determined of SrtA with an inhibitor linked to Cys184 via a disulfide bond (PDB code: $2 \mathrm{MLM} ;{ }^{33}$ Figure $5 \mathrm{~d}$ ).

As in the SrtA-LPAT ${ }^{*}$ analogue, $\mathrm{Ca}^{2+}$ restricts conformation exchange in the thioacyl intermediate, as evidenced by the appearance of ${ }^{15} \mathrm{~N}-\mathrm{HSQC}$ cross-peaks for residues from the $\beta 6 / \beta 7$ loop that are affected by chemical exchange broadening in free SrtA (Figure 1 and Figure S10). ${ }^{26}$ The altered conformation of the $\beta 6 / \beta 7$ loop is interesting, because it forms a connection between the calcium binding site and Cys 184 in the active site. In addition, the thioacyl intermediate also displays a more extended conformation of the $\beta 7 / \beta 8$ loop, which facilitates the nucleophilic attack of the thioester by an oligo-glycine peptide in the next step of the catalytic cycle. ${ }^{21}$

Despite overall structural similarity with the SrtA-LPAT* analogue, there are also significant differences in several segments of the protein (Figure 5 and Figure S9), which correlate with the chemical shift perturbations (Figure S5). Most strikingly, the $\beta 7 / \beta 8$ loop differs in the thioacyl intermediate (Figure $5 b$ ), including the side chains of Trp194 and His120 (Figure 5c). The side chain of Trp194, which resides in the $\beta 7 / \beta 8$ loop, shows a very clear difference in PCSs between free SrtA and the thioacyl intermediate (Figure 1a and b). This residue contributes to the enzyme function, as four-fold decreased enzymatic activity has been reported for the mutant W194A. ${ }^{34}$ The chemical difference between a disulfide bond and a thioester also seems to contribute to structural differences in the $\beta 4 / \alpha 2$ loop (Figure $5 \mathrm{c}$ ), which was proposed to be involved in the subsequent reaction with oligoglycine peptides. ${ }^{21}$

The present work illustrates the power of PCSs for assessing 3D structures of transient enzymatic intermediates present only as minor species in non-equilibrium solutions. To the best of our knowledge, the structure of the short-lived thioacyl intermediate of SrtA determined here with the help of PCSs presents the first structural view, in solution, of an enzymatic intermediate with limited lifetime. The structure determination was made possible by lanthanide tags of carefully tuned reactivity to tag SrtA at a solvent exposed cysteine residue without modifying Cys 184 at the active site. We expect that this strategy will be very useful for the structure analysis of minor protein species that are sufficiently stable to be observable as a separate set of peaks, even if only transiently and with weak intensity.

\section{Acknowledgements a}

We thank J. T. Wang for the synthesis of the T1 tag. X.C.S. thanks Dr. Charles Schwieters for discussions on the use of Xplor-NIH. Financial support by Major National Scientific Research Projects (2013CB910200), Natural Science Foundation of China (21473095 and 21273121), and the Australian Research Council is gratefully acknowledged.

Keywords: protein enzyme $\cdot$ enzyme intermediate $\cdot$ transient protein complex $\cdot$ intermediate structure $\cdot$ NMR spectroscopy

[1] a) K. Henzler-Wildman, D. Kern, Nature 2007, 450, 964-972. b) H. van den Bedem, J. S. Fraser, Nature Methods 2015, 12, 307-318. c) D. D. Boehr, D. McElheny, H. J. Dyson, P. E. Wright, Science 2006, 313, 1638-1642.

[2] a) J. S. Fraser, Acc. Chem. Res. 2015, 48, 423-430. b) A. G. Palmer, Acc Chem. Res. 2015, 48, 457-465. c) P. Hanoian, C. T. Liu, S. Hammes-Schiffer, S. Benkovic, Acc. Chem. Res. 2015, 48, 482-489.

[3] a) P. L. Ramachandran, J. E. Lovett, P. J. Carl, M. Cammarata, J. H. Lee Y. O. Jung, H. Ihee, C. R. Timmel, J. J. Van Thor, J. Am. Chem. Soc. 2011, 133, 9395-9404. b) J. S. Fraser, M. W. Clarkson, S. C. Degnan, R. Erion, D. Kern, T. Alber, Nature 2009, 462, 669-673. c) P. Schanda, V. Forge, B. Brutscher, Proc. Natl. Acad. Sci. USA 2007, 104, 11257-11262. d) R. Neutze, 
K. Moffat, Curr. Opin. Struct. Biol. 2012, 22, 651-659. e) J. Tenboer, S. Basu, N. Zatsepin, K. Pande, D. Milathianaki, M. Frank, M. Hunter, S. Boutet, G. J. Williams, J. E. Koglin, D. Oberthuer, M. Heymann, C. Kupitz, C. Conrad, J. Coe, S. Roy-Chowdhury, U. Weierstall, D. James, D. Wang, T. Grant, A. Barty, O. Yefanov, J. Scales, C. Gati, C. Seuring, V. Srajer, R. Henning, P. Schwander, R. Fromme, A. Ourmazd, K. Moffat, J. J. Van Thor, J. C. Spence, P. Fromme, H. N. Chapman, M. Schmidt, Science 2014, 346, 1242-1246. [4] a) J. L. Battiste, G. Wagner G, Biochemistry 2000, 39, 5355-5365. b) C. Tang, J. Iwahara, M. G. Clore, Nature 2006, 444, 383-386.

[4] L. Banci, I. Bertini, G. G. Savellini, R. Romagnoli, P. Turano, M. A. Cremonini, C. Luchinat, H. B. Gray, Proteins 1997, 29, 68-76.

[5] P. Bradley, K. M. S. Misura, D. Baker, Science 2005, 309, 1868-1871. [6] K. Lindorff-Larsen, R. B. Best, M. A. Depristo, C. M. Dobson, M. Vendruscolo, Nature 2005, 433, 128-132.

[7] Y. Shen, O. Lange, F. Delaglio, P. Rossi, J. M. Aramini, G. Liu, A. Eletsky, Y. Wu, K. K. Singarapu, A. Lemak, A. Ignatchenko, C. H. Arrowsmith, T. Szyperski, G. T. Montelione, D. Baker, A. Bax, Proc. Natl. Acad. Sci. USA 2008, 105, 4685-4690.

[8] P. Neudecker, P. Robustelli, A. Cavalli, P. Walsh, P. Lundström, A. ZarrineAfsar, S. Sharpe, M. Vendruscolo, L. E. Kay, Science 2012, 336, 362-366. [9] C. Camilloni, M. Vendruscolo, Biochemistry 2015, 54, 7470-7476. $[10$.

[11] H. Yagi, K. B. Pilla, A. Maleckis, B. Graham, T. Huber, G. Otting, Structure 2013, 21, 883-890.

[12] Schmitz, C.; Vernon, R.; Otting, G.; Baker, D.; Huber, T. J. Mol. Biol. 2012, 416, 668-677.

[13] Marraffini, L. A.; Dedent, A. C.; Schneewind, O. Microbiol. Mol. Biol. Rev. 2006, 70, 192-221.

[14] K. W. Clancy, J. A. Melvin, D. G. McCafferty, Biopolymers 2010, 94, 385396.

[15] a) A. H. Chan, J. Wereszczynski, B. R. Amer, S. W. Yi, M. E. Jung, J. A. McCammon, R. T. Clubb, Chem. Biol. Drug. Des. 2013, 82, 418-428. b) S. Cascioferro, D. Raffa, B. Maggio, M. V. Raimondi, D. Schillaci, G. Daidone, J. Med. Chem. 2015, 58, 9108-9123.

[16] J. R. Scott, T. C. Barnett, T. C. Annu. Rev. Microbiol. 2006, 60, 397-423. [17] M. W. Popp, H. L. Ploegh, Angew. Chem. Int. Ed. Engl. 2011, 50, 50245032 .
[18] a) J. E. Glasgow, M. L. Salit, J. R. Cochran, J. Am. Chem. Soc. 2016, 138, 7496-7499. b) K. Strijbis, E. Spooner, H. L. Ploegh, Traffic 2012, 13, 780 789. c) H. Hirakawa, S. Ishikawa, T. Nagamune, Biotechnol. J. 2015, 10, 1487-1492

[19] U. Ilangovan, T. Ton-That, J. Iwahara, O. Schneewind, R. T. Clubb, Proc. Natl. Acad. Sci. USA 2001, 98, 6056-6061.

[20] Y. Zong, T. W. Bice, H. Ton-That, O. Schneewind, S. V. Narayana, J. Biol. Chem. 2004, 279, 31383-31389.

[21] N. Suree, C. K. Liew, V. A. Villareal, W. Thieu, E. A. Fadeev, J. J. Clemens, M. E. Jung, R. T. Clubb, J. Biol. Chem. 2009, 284, 24465 [22] S .K. Mazmanian, G. Liu, H. Ton-That, O. Schneewind, Science 1999 285, $760-763$

[23] B. A. Frankel, R. G. Kruger, D. E. Robinson, N. L. Kelleher, D. G. McCafferty, Biochemistry 2005, 44, 11188-11200.

[24] D. C. Cantu, Y. Chen, M. L. Lemons, P. J. Reilly, Nucleic Acids Res. 2011, 39, D342-346.

[25] X. C. Su, T. Huber, N. E. Dixon, G. Otting, Chembiochem. 2006, 7, 15991604.

[26] M. T. Naik, N. Suree, U. llangovan, C. K. Liew, W. Thieu, D. O. Campbell, J. J. Clemens, M. E. Jung, R. T. Clubb, J. Biol. Chem. 2006, 281, 1817-1826. [27] Y. Yang, J. T. Wang, Y. Y. Pei, X. C. Su, Chem. Commun. 2015, 51, 2824-2827.

[28] X. Jia, A. Maleckis, T. Huber, G. Otting, Chem. Eur. J. 2011, 17, 68303836.

[29] C. Schmitz, M. J. Stanton-Cook, X. C. Su, G. Otting, T. Huber, J. Biomol. NMR 2008, 41, 179-189.

[30] C. D. Schwieters, J. J. Kuszewski, N. Tjandra, G. M. Clore, J. Magn. Reson. 2003, 160, 65-73.

[31] C. D. Schwieters, J. Kuszewski, G. M. Clore, Prog. Nucl. Magn. Reson. Spectr. 2006, 48, 47-62.

[32] L. Banci, I. Bertini, G. Cavallaro, A. Giachetti, C. Luchinat, G. Parigi, J. Biomol. NMR 2004, 28, 249

[33] D. Zhulenkovs, Z. Rudevica, K. Jaudzems, M. Turks, A. Leonchiks, Bioorg. Med. Chem. 2014, 22, 5988-6003.

[34] H. Ton-That, S. K. Mazmanian, L. Alksne, O. Schneewind, J. Biol. Chem. 2002, 277, 7447-7752. 
Entry for the Table of Contents (Please choose one layout)

\section{COMMUNICATION}

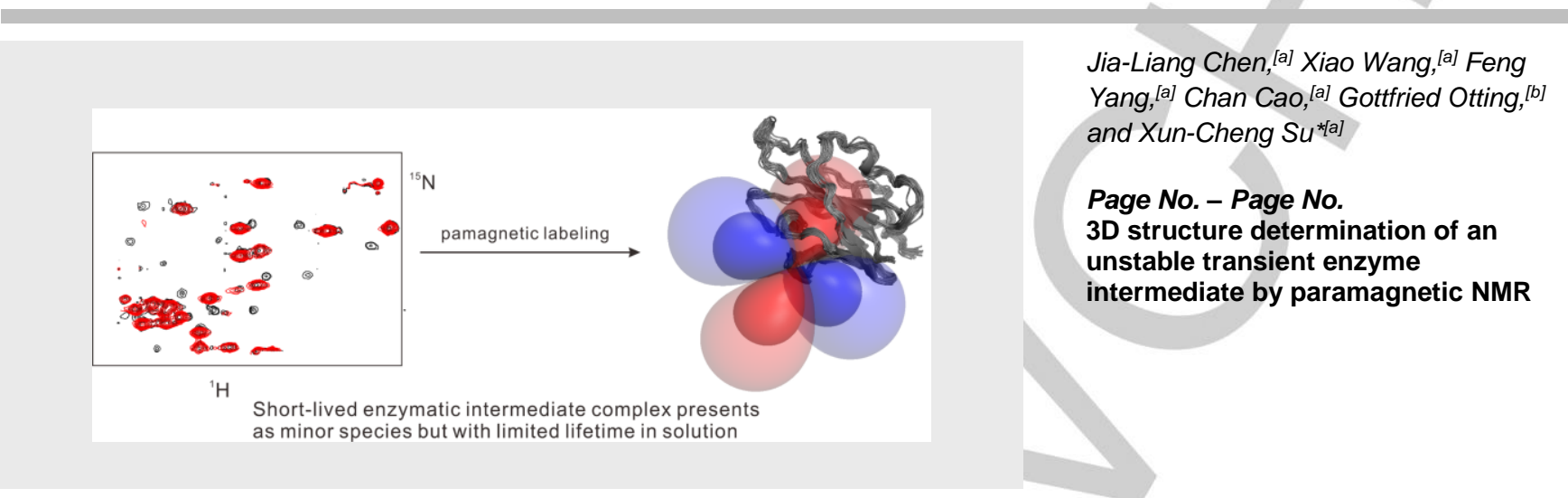

3D structures of low-abundance transient enzyme intermediates can be determined by pseudocontact shifts measured by NMR spectroscopy. The method is demonstrated with the unstable thioacyl intermediate formed by Staphylococcus aureus sortase A and peptide substrate. 